\title{
The perception of verticality and the frame of reference of the visual tilt aftereffect
}

\author{
JOHN J. RIESER \\ Vanderbilt University, Nashville, Tennessee 37203 \\ and \\ MARTIN S. BANKS \\ University of Texas, Austin, Texas 78712
}

\begin{abstract}
Previous research has suggested that the visual tilt aftereffect operates according to a gravitational frame of reference. Three experiments were conducted to test this conclusion further. In each experiment, observers (with head upright) adjusted an illuminated bar to apparent vertical following various adaptation conditions. In Experiment 1, observers were given clear visual cues for objective vertical while adjusting the bar. In Experiment 2, they were not given visual cues for vertical. The adaptation conditions in Experiments 1 and 2 consisted of various combinations of head and stimulus tilt. Experiment 3 investigated the effects of head tilt alone. The results indicated that the tilt aftereffect follows a retinal frame of reference under some conditions (Experiment 1) and appears to follow a gravitational frame under others (Experiment 2). These results can be predicted by a simple model involving two factors, a purely visual aftereffect that follows a retinal frame and an extravisual aftereffect that appears to follow a gravitational frame.
\end{abstract}

When observers tilt their heads laterally, the orientation of the stimulus image relative to the retina changes correspondingly. Nevertheless, the perceived orientation of objects in the environment does not change. This phenomenon of visual orientation constancy implies that the nervous system uses information about head tilt relative to gravity and visual stimulus tilt relative to the retina to determine the perception of orientation (Bishop, 1974). Several experimenters have studied various perceptual phenomena in attempts to determine the level of processing at which orientation constancy is mediated. The results have often been described in terms of frames of reference. For example, Attneave and Olson (1967) and Rock (Rock, 1973; Rock \& Heimer, 1957) have shown that the naming of shapes (whose identity changes with orientation) operates according to a gravitational frame of reference: Observers use the same label across a wide range of lateral head tilts to identify a stimulus whose orientation relative to gravity is constant. Others have found that the McCullough effect (Ellis, 1976), the threshold-elevation aftereffect (Findlay \& Parker, 1972; Mitchell \& Blakemore, 1972), and the oblique

The two authors shared equally in all phases of this research. Order of authorship was decided by the toss of a coin. M.S.B. was supported by NIH Grant HD12572; J.J.R. was supported by NIH Grant HD04510. Reprint requests should be sent to John Rieser, Box 512, Peabody College, Vanderbilt University, Nashville, Tennessee 37203. effect (Banks \& Stolarz, 1975; Lennie, 1974) follow a retinal frame of reference: These visual aftereffects depend on the orientation of stimuli relative to the retina and not relative to gravity. Since these latter effects do not demonstrate orientation constancy, it has been concluded that the mechanisms mediating the effects do not correspond to the mechanisms of visual orientation constancy.

The visual tilt aftereffect (TAE) ${ }^{1}$ may behave differently from those above-mentioned aftereffects. Some evidence indicates that it may operate according to a gravitational frame of reference (Day \& Wade, 1969). Since the current models of the TAE involve relatively simple, peripheral mechanisms (Coltheart, 1971), Day and Wade's findings suggested a somewhat peripheral site for orientation constancy. This paper is focused on the frame of reference of the TAE. To observe the TAE, a subject first views for several minutes a bar or grating tilted 10-20 deg from vertical. Subsequently, a truly vertical test bar or grating appears to be tilted a few degrees in the direction opposite to the original adapting stimulus. The TAE is observed (under standard head-upright conditions) whether the subjects are asked to judge the test stimulus orientation relative to vertical reference lines in the peripheral visual field or relative to their own body axes. As evidence for a gravitational frame of reference, Day and Wade (1969) reported that the TAE depends on the tilt of the adapting and test stimuli relative to gravity rather than to the retina. Unfortunately, 
their procedure did not allow a direct test of gravitational vs. retinal frames (Coltheart \& Cooper, 1972; Wade \& Day, 1973). Moreover, Coltheart and Cooper (1972) showed that the TAE operated according to a retinal frame of reference, at least when gravity cues were made irrelevant by placing the observer in a supine position. Thus, the frame of reference for the TAE has not been investigated under conditions in which both gravitational and retinal frames could operate. Furthermore, the effect of different instructions on the TAE has not been investigated.

We conducted three experiments designed to examine these issues. The TAE was measured in each experiment for different combinations of head tilt and stimulus tilt during adaptation. Generally, four adaptation combinations were presented (tilt is expressed relative to gravity): (1) head vertical and adapting bar vertical $(0 / 0)$; (2) head vertical and adapting bar tilted $(0 / 10)$; (3) head tilted and adapting bar vertical (10/0); and (4) head tilted and adapting bar tilted $(10 / 10)$. Observers always adjusted the test bar to vertical while seated with the head upright. These combinations and the results that would indicate retinal and gravitational frames of reference are shown in Table 1 . Neither a retinal nor a gravitational frame predicts an aftereffect for $0 / 0$, since the adapting bar was not tilted with respect to either. Both frames predict an aftereffect for $0 / 10$ since the adapting bar was tilted relative to both. The interesting conditions are $10 / 0$ and $10 / 10$. The retinal frame predicts an aftereffect for $10 / 0$ because the adapting bar was tilted only relative to the retina. The gravitational frame predicts an aftereffect for $10 / 10$ because the adapting bar was tilted only with respect to gravity. Both the stimulus and subject instructions differed across experiments. In Experiment 1 , subjects were asked to adjust the test bar to parallelism with two vertical reference lines in the peripheral visual field. This experiment measured any strictly visual aftereffect most directly because both the stimulus and the criterion were specified visually. In Experiment 2, subjects were instructed to adjust the test bar to apparent vertical in the

Table 1

Predicted Aftereffects for Retinal and Gravitational Frames of Reference

\begin{tabular}{lcccc} 
& \multicolumn{4}{c}{ Adaptation Condition } \\
\cline { 2 - 5 } \multicolumn{1}{c}{ Frame } & $0 / 0$ & $0 / 10$ & $10 / 0$ & $10 / 10$ \\
\hline Retinal & 0 & + & - & 0 \\
Gravitational & 0 & + & 0 & + \\
\hline
\end{tabular}

Note-Prediction of a clockwise aftereffect is denoted by a " + ," a counterclockwise aftereffect by $a$ " -," and no aftereffect by a " 0 ." The adaptation conditions are described in terms of head tilt followed by stimulus tilt. absence of any visual cues for vertical. Thus, vertical was defined by some internal standard. This task was designed to measure the combined effects of adaptation in the visual and other sensory modalities. In particular, prolonged fixation of a stimulus while the head is tilted could produce a purely visual aftereffect, altering visual judgments of parallelism, and, perhaps, a nonvisual aftereffect, altering the subject's internal standard for apparent vertical. Experiment 3 was very similar to that of Day and Wade (1966) and was designed to measure the contribution of any nonvisual aftereffects following prolonged head tilt alone.

\section{EXPERIMENT 1}

\section{Method}

Apparatus. The stimuli were presented in a modified, twofield tachistoscope. One field contained the adapting line, a $3 \mathrm{deg}$ $\times 6 \mathrm{~min}$ bright bar on an otherwise dark field. The adapting line's orientation (with respect to gravity) was either vertical or 10 deg clockwise from vertical. The other field contained the test line and two reference lines. The test line was also a $3 \mathrm{deg} \times$ $6 \mathrm{~min}$ bright bar. The reference lines were $5 \mathrm{deg} \times 6 \mathrm{~min}$ bright bars, one positioned $5 \mathrm{deg}$ to the left of the test line and the other, 5 deg to the right. Great care was taken to ensure that the stimulus field was otherwise totally dark. The observer could vary the orientation of the test line smoothly by turning a dial. The dial turned a 10-turn potentiometer whose output was registered on a digital voltmeter. Thus, the voltmeter readings indicated the line's orientation relative to gravity.

The observers viewed the stimuli binocularly with natural pupils while seated on an adjustable stool. The position and orientation of the head were stabilized with a dental bite board attached to a metal frame whose orientation could be varied smoothly. Since the frame's center of rotation corresponded to the midpoint between the observer's eyes rather than to the bite board itself, the observer's head could be rotated without changing the vertical or horizontal position of the eyes relative to the stimulus fields. A scale on the frame indicated the orientation of the bite board in degrees from gravitational vertical. The trunk, shoulders, and neck were not constrained, but the observers were encouraged to tilt only the head and not the body during the head-tilted conditions.

Procedure. Three observers were tested-the authors (M.B. and J.R.) and an undergraduate (B.W.) who was unaware of the experimental hypothesis. M.B. was emmetropic; J.R. and B.W. were myopic and wore their optical corrections throughout testing.

Some of the adaptation conditions required lateral head tilt. When the head is tilted, both eyes rotate slightly in the direction opposite to the tilt (Howard \& Templeton, 1966). Consequently, the retina's orientation does not correspond to the head's. In two of our adaptation conditions, a retinal tilt of $10 \mathrm{deg}$ was required. Thus, we needed to determine the amount of head tilt that would yield 10 deg retinal tilt for each observer. We used the following procedure to determine those values. An afterimage of a vertical line was generated, with the observer's head upright. The line was then replaced with a dimmer line tilted 10 deg clockwise. The observer rotated his head (and the bite board) until the afterimage and the tilted line were superimposed. The average of three such adjustments was then used as the amount of head tilt needed for that observer.

An experimental session was conducted in the following manner. First, the observer dark adapted for a few minutes. The observer then gave three measurements of perceived vertical 
by adjusting the test line until it appeared parallel to the reference lines. Fixation was always on the test line. After each adjustment, the experimenter changed the test line's orientation by at least $5 \mathrm{deg}$, alternating the direction of change from trial to trial.

Following this, one of four adaptation conditions was presented (see Table 1). In each of these conditions, the observer fixated the appropriate adapting line for $3 \mathrm{~min}$. Following this adaptation period, the observer, with his head vertical, adjusted the test line to parallelism with the reference lines. Transition from the adapting condition to this testing situation required about $5 \mathrm{sec}$, during which the observer closed his eyes. Once the testing situation was set up, however, the observer maintained fixation on the test line. It generally required 5 to $10 \mathrm{sec}$ for the observer to adjust the test line to his satisfaction. Three such sequencesadaptation followed by test line adjustment-were completed for a condition within an experimental session. The magnitude of the tilt aftereffect was the difference in orientation between the preadaptation and postadaptation test line adjustments.

Since the TAE is quite persistent, an observer did not begin another adaptation condition until at least $1 \mathrm{~h}$ had passed after completing the last one. Each observer eventually contributed two sets of three measurements for each adaptation condition.

\section{Results}

The results of Experiment 1 are summarized in Figure 1. The separate panels, labeled MB, JR, and BW, display individual observer's TAE magnitudes for each of the four adaptation conditions. The panel labeled MEAN displays the group averages. The brackets indicate standard errors. Positive values on the abscissa refer to clockwise TAEs: That is, the observers' postadaptation test line judgments differed in the clockwise direction from their preadaptation adjustments. Negative abscissa values refer to counterclockwise aftereffects. Large TAEs were not observed for the $0 / 0$ condition, a result that is consistent with both the retinal and gravitational frame hypotheses (see Table 1). A significant positive aftereffect was obtained for the $0 / 10$ condition, an outcome that is also consistent with both hypotheses. The head-tilted conditions, 10/0 and $10 / 10$, were the important ones for testing the two hypotheses. Large TAEs were not observed for the $10 / 10$ condition. This finding is consistent with the retinal frame hypothesis; since the adapting line was vertical relative to the retina, this hypothesis predicts no aftereffect. The finding is, of course, inconsistent with the gravitational frame point of view; the adapting line was tilted clockwise with respect to gravitational vertical, so a positive aftereffect is predicted. The figures exhibit a large negative aftereffect for the $10 / 0$ condition. This result is also consistent with the retinal hypothesis because the adapting line was tilted counterclockwise relative to the retina. The gravitational view fails again; the adapting line was vertical with respect to gravity, and, consequently, that hypothesis predicts no aftereffect.

We performed an analysis of variance to substantiate the correspondence between the data and the predictions of the retinal frame hypothesis.

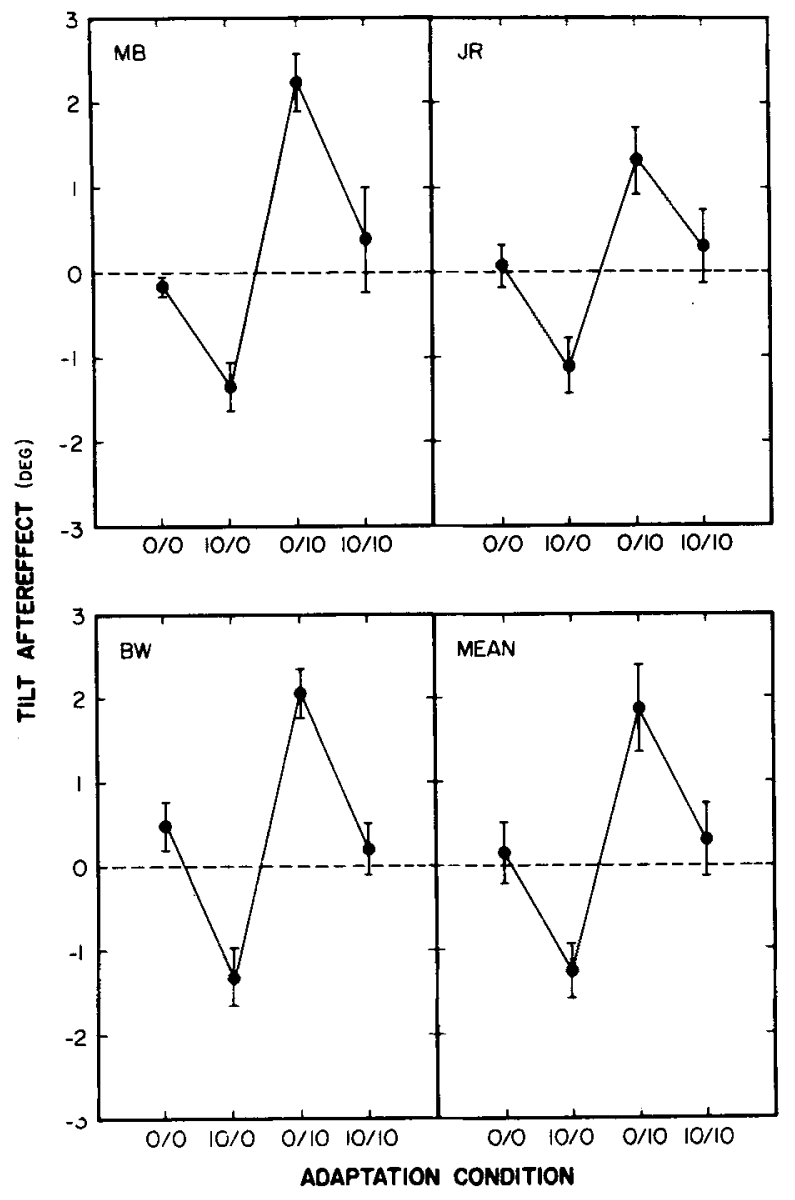

Figure 1. Results from Experiment 1, in which judgments of vertical were based on visible reference lines. For each subject, the magnitude of the tilt aftereffect is plotted as a function of head tilt and stimulus tilt. The brackets around each point indicate standard errors.

Head tilt (re gravity) and adapting line tilt (re retina) were treated as within-subjects factors. The main effect of adapting line was significant $[F(1,2)=$ $52.63, \mathrm{p}=.018]$. No other main effects or interactions were significant. This indicates that the adapting line's orientation relative to the retina was the most significant determinant of aftereffect magnitude. We performed another analysis of variance with head tilt and adapting line tilt treated as within-subjects factors, but this time with adapting line tilt expressed relative to gravity. Neither the adapting line tilt $[F(1,2)=2.07, p=.287]$ nor the head tilt $[F(1,2)=5.23, p=.149]$ factors were significant. The Line Tilt by Head Tilt interaction was significant $[F(1,2)=52.63, p=.018]$. These results imply that the tilt of the adapting line with respect to gravity was not a significant determinant of aftereffect magnitude.

In summary, the results of Experiment 1 were entirely consistent with the retinal frame hypothesis. 


\section{EXPERIMENT 2}

Whereas Experiment 1 was designed to test for purely visual effects in the TAE, Experiment 2 was designed to examine possible nonvisual contributions to the aftereffect. To this end, we eliminated all visual information for vertical during test line adjustment by deleting the vertical reference lines of Experiment 1. Otherwise, Experiments 1 and 2 were identical.

\section{Method}

Apparatus and Procedure. The stimulus-generating and response-recording equipment were the same as in Experiment 1 except that the vertical reference lines in the test field were eliminated. Thus, the test field appeared totally dark (even after 30 min of dark adaptation) except for the test line. A shroud was placed near the observer's head to mask any features on the tachistoscope or other equipment that might provide environmental information.

The procedure was identical to that of Experiment 1 except that the observer always adjusted the test line to apparent gravitational vertical rather than to parallelism with the reference lines. Once again, four adaptation conditions were presented $-0 / 0,0 / 10$, $10 / 0$, and $10 / 10$. Each observer completed six trials for each of the conditions, but, again, only three trials were presented in a single session.

\section{Results}

Figure 2 shows individual and group average TAE magnitudes for the four adapting conditions. The brackets indicate standard errors. Note that the variability was somewhat higher than in Experiment 1 , possibly because the absence of reference lines made the criterion more uncertain. As expected, no aftereffect was evident in the $0 / 0$ condition, and a positive aftereffect was observed in the $0 / 10$ condition. Interestingly, the critical head-tilted conditions produced results that were very different from those of Experiment 1. A significant positive aftereffect was obtained for the $10 / 10$ condition. Such a result is consistent with the gravitational hypothesis, since the adapting line was tilted clockwise relative to gravity. It is not consistent with the retinal hypothesis, because the adapting line was not tilted relative to the retina. Moreover, no aftereffect was observed in the $10 / 0$ condition, a finding that is also consistent with the gravitational point of view since the adapting line was not tilted with respect to gravity. In short, the head-tilted conditions produced quite disparate results between Experiments 1 and 2. The results of Experiment 2 seemed consistent with the gravitational hypothesis.

The results of analyses of variance conducted on these data support this view. We treated head tilt (re gravity) and line tilt (re gravity) as within-subjects factors. The main effect of line tilt was significant $[F(1,2)=20.69, p=.045]$. No other main effects or interactions were significant. Thus, line tilt with respect to gravity was a significant determinant of

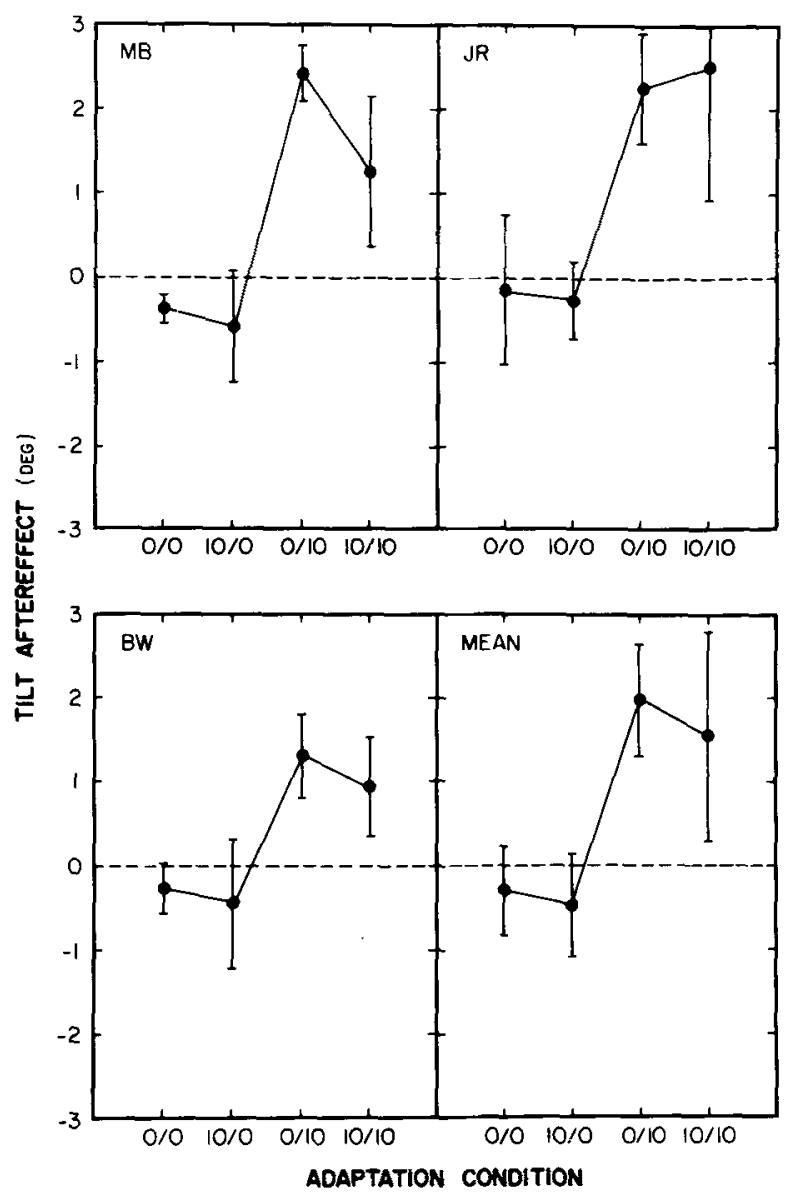

Figure 2. Results from Experiment 2, in which judgments of apparent vertical were made without reference lines. For each subject, the magnitude of the tilt aftereffect is plotted as a function of head tilt and stimulus tilt. The brackets around each point indicate standard errors.

aftereffect magnitude. When a similar analysis was conducted-but, in this case, with line tilt expressed relative to the retina-no significant main effects emerged [for head tilt, $F(1,2)=1.74, p=.317$; for line tilt, $F(1,2)=3.96, p=.185]$. The Head Tilt by Line Tilt interaction was significant, as expected $[F(1,2)=20.69, p=.045]$.

\section{EXPERIMENT 3}

In Experiments 1 and 2, the behavior of the TAE depended on whether or not a visual reference to vertical was present. With reference lines, it appeared to follow a retinal frame of reference, and without them, a gravitational frame.

At least two factors can be involved in judgments of vertical following prolonged head tilt and inspection of an adapting line: (1) the visually induced tilt aftereffect that occurs with disparities between adapting line and test line orientations, and (2) after- 
Table 2

Magnitude of Tilt Aftereffect (in Degrees) in Experiment 3: Mean and Standard Deviation

\begin{tabular}{cccccc}
\hline & \multicolumn{2}{c}{$10 /$ NS with Lines } & & \multicolumn{2}{c}{ NS without Lines } \\
\cline { 2 - 3 } \cline { 5 - 6 } Observer & Mean & SD & & Mean & SD \\
\hline M.B. & +.32 & .24 & +1.80 & .38 \\
J.R. & -.16 & .33 & +1.54 & .74 \\
B.W. & -.12 & .21 & +1.24 & .46 \\
Mean & +.01 & .34 & +1.53 & .57 \\
\hline
\end{tabular}

effects that occur after prolonged tilting of the head and/or body involving extravisual cues from the upper three vertebrae and/or the vestibular system (Howard \& Templeton, 1966). To understand the impact of these two factors on our results, we attempted to isolate them experimentally. In Experiment 3, we deleted the adapting line altogether in order to measure the influence of proprioceptive and/or vestibular aftereffects alone. This experiment is very similar to those of Day and Wade (1966, 1968).

\section{Method}

Apparatus and Procedure. The same apparatus was used again. The stimulus field during adaptation, however, was always totally dark. The stimulus field and the observer's peripheral visual field were masked to ensure that no extraneous visual cues were present. The test field consisted of the same adjustable test line as before. In some cases, the reference lines were present; in others, they were not.

The experiment was identical procedurally to the first two except that only two adaptation conditions were presented. In one condition, 10/NS with reference lines, the observer adjusted the test line to parallelism with the reference lines both before and after adaptation. During adaptation, the observer, with head tilted clockwise, viewed the empty stimulus field for $3 \mathrm{~min}$. He was then returned to an upright position and adjusted the test line. The second condition, 10/NS without reference lines, was identical to the first except that no reference lines were presented during pre- or postadaptation adjustment to vertical.

\section{Results}

Table 2 summarizes the results of Experiment 3. The magnitude of the TAE in degrees is shown for the two adaptation conditions. In the $10 / \mathrm{NS}$ with reference lines condition, the TAE was not significantly different from zero $[\mathrm{t}(2)=.07, \mathrm{p}=.94]$. In the $10 /$ NS without reference lines condition, however, the aftereffect was significantly greater than zero $[\mathrm{t}(2)=9.53, \mathrm{p}=.011]$; that is, a positive aftereffect was obtained. This second finding is consistent with Day and Wade's $(1966,1968)$ observations. Thus, a positive TAE occurred after lateral head tilt alone, as long as no reference lines were present during test line adjustment. No aftereffect was observed when the reference lines were added.

\section{DISCUSSION}

In this discussion, we consider three questions.
First, how do our findings match those from previous experiments? Second, how can one account for the similarities and dissimilarities in the results of our Experiments 1, 2, and 3? Third, how do our findings relate to the original question concerning perception of vertical and the mechanisms of visual orientation constancy?

Some of the conditions in our experiments are conceptually analogous to those of previous experiments. In such cases, the results are consistent with one another. For example, our $0 / 10$ condition with reference lines is the same as some of Gibson and Radner's (1937) and Parker's (1972) conditions. Although the size and type of stimuli varied across these experiments, the average magnitudes of the TAE across the experiments were similar. Our 10/NS condition without reference lines is analogous to Day and Wade's (1966) experiment, and the aftereffect magnitudes were similar. (The magnitudes in our experiments were actually slightly greater, but that was probably because we tilted the subjects' heads more than $10 \mathrm{deg}$ in order to attain a retinal tilt of $10 \mathrm{deg}$; Day and Wade simply tilted the head 10 deg.)

Other experiments have presented conditions that appeared similar to ours, but were actually dissimilar in important ways. As mentioned earlier, Day and Wade (1969) reported data supporting a gravitational frame hypothesis, but their procedure did not allow differential gravitational and retinal frame predictions (Coltheart \& Cooper, 1972; Wade \& Day, 1973). Coltheart and Cooper's experiment (1972) suggested that the TAE follows a retinal frame, but their procedure did not allow any gravitational effects to emerge: Their observers were supine throughout adaptation and testing, and the stimulus and head were tilted in a direction orthogonal to gravitational vertical. Thus, despite the fact that Day and Wade's results are similar to our Experiment 2 results, and Coltheart and Cooper's are similar to those of Experiment 1, their experiments are logically dissimilar from ours, and the results, consequently, should not be compared directly.

We next consider how one might account for the pattern of results in our experiments. To this end, a simple model of the TAE and the effects of lateral head tilt is proposed. The model states that two aftereffects can be involved: (1) a strictly visual tilt aftereffect (VTAE), and (2) a neck proprioceptive and/or vestibular aftereffect (NPAE).

According to this model, the VTAE occurs whenever there is an orientational difference between the adapting and test lines relative to a retinal frame of reference (Coltheart \& Cooper, 1972; Mitchell \& Muir, 1976). Inspection of the adapting line causes a shift of the apparent orientation of the test line away from the adapting line's orientation. Again, this shift occurs relative to retinal orientation. The 
VTAE occurs whether or not reference lines are present during test line adjustment.

The NPAE occurs when the head is tilted laterally relative to gravity (Day \& Wade, 1966). It is independent of the orientation (or even the presence) of the adapting line. The NPAE causes a shift of the apparent orientation of the head, when it is returned to upright, away from the direction in which the head was tilted during adaptation. Because of this, observers perceive the test line as tilted away from vertical in the same direction in which they perceive their heads to be tilted. Without visual reference lines to correct this error, they adjust the test line to compensate for the apparent tilt. In our experiments, this would emerge as a positive aftereffect (i.e., observers would adjust the test line clockwise from true vertical) because head tilt was always clockwise in the head-tilted adaptation conditions. The predicted positive aftereffect is illustrated by the 10/NS condition without reference lines of Experiment 3. In contrast, when reference lines are provided and the observer is asked to adjust the test line to parallelism with those lines, the effects of the NPAE are not manifest. This is illustrated by the 10/NS condition with reference lines of Experiment 3.

These assumptions are summarized in Table 3, which shows how the VTAE and NPAE should affect test line adjustments in all of the conditions of our experiments. We have indicated conditions in which no effect is predicted by a " 0 ," conditions in which a positive aftereffect is predicted by a " +," and conditions in which a negative aftereffect is predicted by a " - ." The upper half of the table shows predictions for cases in which reference lines were present during test line adjusting. No VTAE is predicted for $0 / 0$ or $10 / 10$ because, in both cases, the adapting line was upright relative to the retina. Neither is a VTAE predicted for $10 / \mathrm{NS}$, since no adapting line was presented. We predict a positive VTAE for $0 / 10$, because the adapting line was tilted clockwise relative to the retina, and a negative VTAE for $10 / 0$, since the adapting line was tilted counterclockwise relative to the retina. No effect of the NPAE is expected for any of the conditions because visual reference lines were provided. If we make the simple assumption that the effects of the two aftereffects add, the pattern of results shown in the row labeled "sum" are predicted. One can see from Figure 3 and Table 2 that the actual results followed this pattern quite accurately.

The lower half of Table 3 displays the predictions when no visual reference lines were provided during test line adjustment. The VTAE should behave in the same manner described above since its effect does not depend on the presence of reference lines. The effect of the NPAE should, however, be quite different. Of course, no NPAE is expected for the $0 / 0$ and $0 / 10$ conditions since they did not involve head tilt; but positive aftereffects are predicted for $10 / 0,10 / 10$, and $10 /$ NS because the head was tilted clockwise in all of those conditions. The addition of the VTAE and NPAE effects yields the pattern of results shown in the last row of the table. This pattern corresponds very well with the results of Experiment 2 (Figure 2) and Experiment 3 (Table 2).

The final question of interest concerns the relation between our data and the two-factor model on the one hand, and the more general phenomena of visual orientation constancy and perception of vertical on the other. Taken alone, the responses in Experiment 2 indicate that the VTAE exhibits orientation constancy. However, our model indicates that two factors, aftereffects opposite in direction but similar in magnitude, operated to produce these results. Nevertheless, it is interesting to consider the potential importance of such opposite processes in everyday perception. The most important condition of Experiment 2 is the $10 / 0$ condition because, we believe, it most closely reveals a useful perceptual process related to, but different from, visual orientation constancy. To understand this, consider the three phases of the 10/0 condition. First, the observer, with head upright, makes judgments of the perceived vertical. The head is then tilted laterally and the observer views an objective vertical line. Finally,

Table 3

Aftereffects Predicted From the Two-Factor Model

\begin{tabular}{|c|c|c|c|c|c|c|c|c|c|c|}
\hline & \multicolumn{10}{|c|}{ Adaptation Condition } \\
\hline & \multicolumn{5}{|c|}{ With Reference Lines } & \multicolumn{5}{|c|}{ Without Reference Lines } \\
\hline & $0 / 10$ & $0 / 10$ & $10 / 0$ & $10 / 10$ & $10 / \mathrm{NS}$ & $0 / 0$ & $0 / 10$ & $10 / 0$ & $10 / 0$ & $10 / \mathrm{NS}$ \\
\hline VTAE & 0 & + & - & $\mathbf{0}$ & 0 & 0 & + & - & 0 & 0 \\
\hline NPAE & 0 & 0 & 0 & 0 & 0 & 0 & 0 & + & + & + \\
\hline SUM & 0 & + & - & $\mathbf{0}$ & 0 & 0 & + & 0 & + & + \\
\hline
\end{tabular}

Note-Prediction of a clockwise aftereffect is denoted by a "+," a counterclockwise aftereffect by a "-," and no aftereffect by a " $0 . "$ The adaptation conditions are described in terms of head tilt followed by stimulus tilt. "NS" identifies those conditions in which there was no visual stimulus during adaptation. 


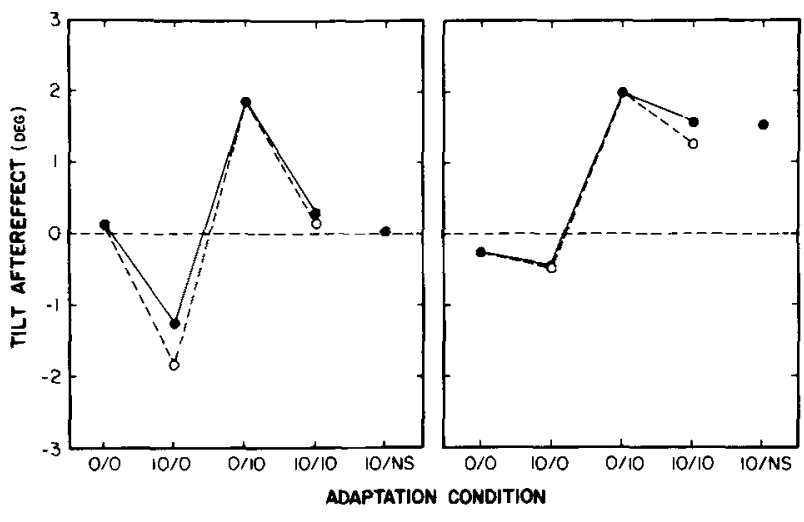

Figure 3. The fits between predictions derived from the twofactor model and the observed tilt aftereffects are displayed for Experiment 1 (on the left side of the figure) and Experiment 2 (on the right side). The dark circles represent the means of the three subjects' tilt aftereffects in the different conditions defined in terms of head tilt and stimulus tilt. The light circles represent predictions from the two-factor model. These estimates were drawn from the results of Experiment 1 (VTAE) and Experiment 3 (NPAE).

the head is returned to upright and the observer makes another judgment of verticality. The preadaptation and postadaptation judgments do not differ (see Figure 2). This experimental procedure is quite similar to everyday situations in which one views a visual scene with head upright, then tilted, and then upright again. As in our experiment, the pretilt and posttilt perceptions of the scene's orientation do not differ. The $10 / 0$ condition in Experiment 2 is more relevant here than that condition in Experiment 1. We believe the conditions of Experiment 1 are not relevant to the everyday situations we are discussing because the visual information presented differs greatly between adaptation and testing. During adaptation, only the adapting line is present. During testing, two objectively vertical reference lines are added. In the everyday situation discussed here, the visual information does not change from head-tilted to head-upright viewing. Thus, the 10/0 condition in Experiment 2 is relevant because it, too, involves no change in the visual information presented. Another relevant experimental condition would be to present constant reference lines during testing and adaptation, mimicking the everyday situation in which visual cues for vertical are present whether the head is tilted or not. If such a condition were run, we believe the results would be similar to those of the $10 / 0$ condition of Experiment 2; the VTAE would affect both the test and reference lines (assuming that the VTAE is observed with off-foveal presentation), and, therefore, any line that appeared to be parallel to the reference lines before adaptation would also appear parallel after adaptation. Furthermore, no shift of the perceived orientation of the lines relative to the internal standard would be expected because of the compensating effects of the VTAE and NPAE.

This seems to be a useful perceptual constancy related to visual orientation constancy. However, for such a constancy to obtain generally, the magnitudes of the VTAE and NPAE would have to be similar across some range of tilts, and the effects would have to vary in magnitude in the same manner for different sizes, shapes, and contrasts in visual scenes. We know of no experimental data comparing the VTAE and NPAE on the same subjects for various tilt magnitudes and stimulus parameters. Nonetheless, it is interesting to note that the NPAE increases linearly with amount of head tilt up to 20 and perhaps even 30 deg (Day \& Wade, 1966). Similarly, the magnitude of the VTAE increases linearly with amount of stimulus tilt up to about $10 \mathrm{deg}$ (Campbell \& Maffei, 1970; Gibson \& Radner, 1937; Mitchell \& Muir, 1976). It is also interesting to note that the magnitude of the VTAE is not influenced by a variety of stimulus parameters such as size and contrast, as long as the parameters are similar in the adapting and test stimuli (Parker, 1972). Presumably, the NPAE is also uninfluenced by stimulus size and contrast since it does not require a visual stimulus during adaptation and involves a shift in internal standard. It is possible, therefore, that the conditions for this kind of perceptual constancy are met for head tilts of $10 \mathrm{deg}$ but that the mechanisms would break down for greater tilts. Thus, the VTAE and NPAE may work together to provide stable perception of orientation for relatively small head tilts.

\section{REFERENCES}

Attneave, F., \& Olson, R. K. Discriminability of stimuli varying in physical and retinal orientation. Journal of Experimental Psychology, 1967, 74, 149-157.

Banks, M.S., \& Stolarz, S. J. The effects of head tilt on meridional differences in acuity: Implications for orientation constancy. Perception \& Psychophysics, 1975, 17, 17-22.

Bishop, N. Optic-vestibular orientation to the vertical. In $\mathbf{H}$. Kornhuber (Ed.), Handbook of sensory physiology, VI/2. New York: Springer-Verlag, 1974.

Campbelt, F. W., \& M AfFe I, L. Electrophysiological evidence for the existence of orientation and size detectors in the human visual system. Journal of Physiology, 1970, 207, 635-652.

Coltheart, M. Visual feature analyzers and aftereffects of tilt and curvature. Psychological Review, 1971, 79, 114-121.

Coltheart, M., \& Cooper, C. M. The retinal reference of the tilt aftereffect. Perception \& Psychophysics, 1972, 11, 321-324.

DAY, R. H., \& WADE, N. J. Visual spatial aftereffect from prolonged head tilt. Science, 1966, 154, 1201-1202.

DAY, R. H., \& WADE, N. J. Involvement of neck proprioceptive system in visual aftereffect from prolonged head tilt. Quarterly Journal of Experimental Psychology, 1968, 20, 290-293.

DAY, R. H., \& WADE, N. J. The reference for visual normalization. American Journal of Psychology, 1969, 82, 191-197.

Ellis, S. R. Orientation constancy of the McCollough effect. Perception \& Psychophysics, 1976, 19, 183-192. 
Findlay, J. M., \& Parker, D. M. An investigation of visual orientation constancy using orientation-specific properties of acuity and adaptation. Perception, 1972, 1, 305-313.

Gibson, J. J., \& RAdNER, M. Adaptation, aftereffect and contrast in the perception of tilted lines. Journal of Experimental Psychology, 1937, 20, 453-467.

How ARD, I. P., \& Templeton, W. B. Human spatial orientation. New York: Wiley, 1966.

LENNIE, P. Head orientation \& meridional variations in acuity. Vision Research, 1974, 14, 107-111.

Mitchell, D., \& Blakemore, L. The site of orientation constancy. Perception, 1972, 1, 315-320.

Mitchell, D., \& Muir, D. W. Does the tilt aftereffect occur in the oblique meridian? Vision Research, 1976, 16, 609-614.

Parker, D. M. Contrast and size variables and tilt aftereffect. Quarterly Journal of Experimental Psychology, 1972, 24, 1-7.

Rock, I. Orientation and form. New York: Academic Press, 1973.

Rock, I., \& Heimer, W. The effect of retinal and phenomenal orientation on the perception of form. American Journal of Psychology, 1957, 10, 493-511.

WADE, N. J., \& DAY, R. H. A correction to "The retinal reference of the tilt aftereffect" by Coltheart and Cooper. Perception \& Psychophysics, 1973, 13, 319-320.

\section{NOTE}

1. The tilt aftereffect following inspection of a tilted visual stimulus is properly considered a visual tilt aftereffect. However, in some conditions of these experiments, aftereffects of visual stimulation together with proprioceptive stimulation were measured. For this reason, the measured aftereffects are referred to simply as tilt aftereffects (TAE) throughout most of this paper.

(Received for publication June 6, 1980; revision accepted October 26,1980 .) 